\title{
Artigo Revisão
}

\section{Detecção e tratamento das complicações crônicas do diabetes melito: Consenso da Sociedade Brasileira de Diabetes e Consel ho Brasileiro de Oftalmologia}

\author{
J .L. Gross*, M. NehME** \\ *Sociedade Brasileira de Diabetes; **Conselho Brasileiro de Oftalmologia.
}

UNITERMOS: Retinopatia. Neuropatia. Nefropatia. Diabetes Melito.

KEY WORDS: Retinopathy. Neuropathy. Nephropathy. Diabetes Mellitus.

\section{INTRODUÇÃO}

O diabetes meli to (DM) acomete cerca de 7,6\% da população brasileira entre 30 e 69 anos de idade. Cerca de $50 \%$ dos pacientes desconhecem o diagnóstico e $24 \%$ dos pacientes reconhecidamente portadores de DM não fazem qualquer tipo de tratamento 1 .

As compl icações crôni cas do diabetes mel ito (DM) são as principais responsáveis pela morbidade e mortalidade dos pacientes diabéticos. As doenças cardiovasculares representam a principal causa de morte (52\%) em pacientes diabéticos do tipo $2^{2}$. Diversos fatores de risco, passíveis de intervenção, estão associados ao maior comprometimento cardiovascular observado nos pacientes diabéticos. Entre eles estão a presença da Nefropatia Diabética (ND) e da Hipertensão Arterial Sistêmica (HAS).

O impacto desfavorável da HAS e das dislipidemi as sobre a mor bimortalidade cardiovascular é amplamente reconhecido, bem com a freqüente associ ação destas condi ções ao DM . Dados da Organização Mundial da Saúde mostram significativa el evação da mortalidade de indivíduos com DM tipo 1 e 2 na presença de $\mathrm{HAS}^{3}$. Por outro lado, são numerosas as evi dências de que o tratamento anti hipertensivo é capaz de reduzir a incidência de eventos cardiovasculares em indivíduos com e sem DM ${ }^{4,5}$. Também a intervenção sobre a dislipidemia tem se mostrado benéfica no controle da doença macrovascular de indivíduos diabéti $\cos ^{6}$.

A ND acomete cerca de $40 \%$ dos pacientes diabéticos eéa principal causa de insuficiência renal em pacientes que ingressam em programas de diálise. A mortalidade dos pacientes diabéticos em programas de hemodiálise é maior do que a dos não- diabéticos. Cerca de $40 \%$ dos pacientes morrem no primeiro ano de tratamento, principalmente por doença cardiovascular ${ }^{7}$. O custo do tratamento da insuficiência renal crônica (IRC) é elevado. De acordo com os dados obti dos junto à Secretaria de Saúde do Meio Ambiente do E stado do Rio Grande do Sul, o custo direto de um paciente em hemodiálise por ano é de $R \$ 13.902,00$ sem contar o acesso vascular, as medicações ( $R \$ 4.000,00)$, as eventuais hospitalizações e as consultas. Esse tipo de tratamento consome cerca de $7 \%$ do total disponível para a assistência médica deste E stado.

A ND apresenta uma fase inicial, denominada de nefropatia incipiente (fase de microalbuminúria) e uma fase mais avançada definida como nefropatia clínica (fase de macroalbuminúria). Existem diversas estratégias terapêuticas que podem ser empregadas para reverter as alterações encontradas na fase de mi croal bumi núria e retardar a evolução da fase de macroal buminúria para a IRC. A ND pode ser diagnosticada precocemente pela medida da al buminúria. Atual mente já estão bem definidos procedimentos simples e efetivos para realizar orastreamento9,10. É possível a redução de custos utilizando-se uma abor dagem inicial simplificada para o rastreamento. A medida de proteinúria e albuminúria custam $\mathrm{R} \$ 0,17$ e $\mathrm{R} \$$ 0,78 , respectivamente.

E studos real izados em países da E uropa demonstraram a el evada rel ação benefício/custo do emprego de medidas de prevenção da ND ${ }^{11}$. No entanto, nestes países em menos de $50 \%$ dos pacientes com diabetes do tipo 2 são realizados testes de rastreamento para a ND ${ }^{12}$.

A Retinopatia Diabética (RD) acomete cerca de $40 \%$ dos pacientes diabéti cos e é a principal causa de cegueira em pacientes entre 25 e 74 anos $^{13}$. A mai oria dos casos de ceguei ra (90\%) érelacionada à RD e pode ser evitada através de medidas adequadas, que incluem, além do controle da gl i cemi a e da pressão arterial, a realização do diagnóstico em uma fase inicial e passível de intervenção. Essas medidas diminuem a progressão das alterações 


\begin{tabular}{|c|c|c|}
\hline Categoria & Urina de 24 horas* & Amostra de urina** \\
\hline \multicolumn{3}{|l|}{ Normal: } \\
\hline Normoalbuminúria & $<20 \mu \mathrm{g} / \mathrm{min}$ & $<17 \mathrm{mg} / \mathrm{L}$ \\
\hline \multicolumn{3}{|l|}{ Nefropatia Incipiente: } \\
\hline Microalbuminúria & 20 a $200 \mu \mathrm{g} / \mathrm{min}$ & 17 a $174 \mathrm{mg} / \mathrm{L}$ \\
\hline \multicolumn{3}{|l|}{ Nefropatia Clínica: } \\
\hline Macroalminúria & $>200 \mu \mathrm{g} / \mathrm{min}$ & $>174 \mathrm{mg} / \mathrm{L}$ \\
\hline Proteinúria & $\geq 500 \mathrm{mg} / 24 \mathrm{~h}$ & $\geq 430 \mathrm{mg} / \mathrm{L}$ \\
\hline \multicolumn{3}{|c|}{$\begin{array}{l}\text { * American Diabetes Association. Diabetes Care } 21 \text { (suppl I): } \\
\text { S50-S53, } 1998 \\
\text { ** Zelmanovitz T et al. Diabetes Care } 20: 516-519,1997 \\
\text { Zelmanovitz T et al. Diabetes Care 21: 1076-1079, } 1998.0\end{array}$} \\
\hline
\end{tabular}

Quadro 2 - Instruções para aplicação do monofilamento de nylon

1. Mostrar o filamento ao paciente e aplicá-lo em sua mão para que ele possa reconhecer o tipo de estímulo e "perca o medo".

2. Pedir que o paciente não olhe para o local que estará sendo testado.

3. Pedir que o paciente preste atenção e simplesmente responda "sim" quando sentir o filamento; não perguntar se ele sente ou não, para não induzi-lo.

4. Ao aplicar o filamento, mantenha-o perpendicularmente à superfície testada, a uma distância de $1-2 \mathrm{~cm}$ : com um movimento suave, faça-o curvar-se sobre a pele e retire o, contando mentalmente a seqüência numérica "10011002 " enquanto o filamento toca a pele, curva-se e sai do contato.

5. Não use movimentos bruscos na aplicação; se o filamento escorregar pelo lado, desconsidere a eventual resposta do paciente e teste o mesmo local novamente mais tarde.

6. Use uma seqüência ao acaso nos locais de teste, para não induzir o paciente a prever o local seguinte onde o filamento será aplicado.

7. Havendo áreas ulceradas, necróticas, cicatriciais ou hiperceratóticas, teste o perímetro da mesma, e não sobre a lesão.

8. Se o paciente não responder à aplicação do filamento num determinado local, continue a seqüência randômica e volter posteriormente àquele local para confirmar.

9. Anote os resultados segundo a percepção do filamento em cada região testada.

10. Conserve o filamento protegido, cuidando para não amassá-lo ou quebrá-lo, se necessário, limpe-o com solução de hipoclorito de sódio a 1:10.

retinianas, não revertendo os danos já estabelecidos. Portanto, éimperativo que seja feito o diagnóstico da RD em suas fases iniciais antes que lesões que comprometem a visão tenham ocorrido. 0 mel hor procedimento para realizar a detecção precoce da RD ainda não foi determinado. I dealmente, o rastreamento para RD deveria ser realizado por oftalmologista da rede pública, mas este sistema tem se mostrado insuficiente em locais como o

\begin{tabular}{|c|c|}
\hline $\begin{array}{l}\text { Quadro } 3 \text { - Rastreamento do pé em } \\
\text { Qualquer achado positivo (SIM) identifica } \\
\text { em risco }\end{array}$ & $\begin{array}{l}\text { e úlcera. } \\
\text { te com pé }\end{array}$ \\
\hline Ulcera prévia & sim/não \\
\hline Neuropatia & \\
\hline Monofilamento & sim/não \\
\hline Calosidade & sim/não \\
\hline Deformidade & sim/não \\
\hline Calçados inadequados & sim/não \\
\hline Doença vascular periférica & \\
\hline Ausência de pulsos tibial posterior/pedioso & sim/não \\
\hline Micose, Bolha, Rachaduras, Fissuras & sim/não \\
\hline
\end{tabular}

Pacientes que apresentam calosidades sangrantes, úlceras com celulite e/ou abscesso, deformidades importantes (ex. artropatia de Charcot), claudicação intermitente e sinais de isquemia requerem encaminhamento imediato para equipe especializada.

ReinoU nido, na medida em queapenas uma pequena porção dos pacientes tem acesso ao especialista ${ }^{14}$. I magens da retina obtidas em sistemas digitais podem ser visualizadas em um monitor de computador e armazenadas em discos. Esse exame pode ser efetuado por técnicos e posteriormente analisado por oftalmologistas ${ }^{15}$.

Dados epidemiológicos brasileiros indicam que as amputações de membros inferi ores ocorrem 100 vezes mais freqüentemente em pacientes com $D M^{16}$. Pacientes diabéticos com lesões graves nos pés constituem $51 \%$ dos pacientes internados em enfermarias dos Serviços de Endocrinologia nos Hospitais Universitários, com duração que pode chegar a 90 dias $^{17}$. Calculou-se que no Hospital de Clíni cas de Porto Alegre uma internação de 21 dias para tratamento de úlcera nos pés de um paciente diabético custa cerca de $\mathrm{R} \$ 7.000,00$. Em nível ambulatorial, o custo trimestral, incluindo apenas a distri buição de anti bióticos orais e antimicóticos orais e tópicos, atingiu $\mathrm{R} \$ 31.400,00$ no Centro de Pé Diabético da Fundação Hospitalar do Distrito F ederal ${ }^{18}$. A grande maioria (85\%) dos casos graves que necessita internação hospital ar, origina-se de úlceras superficiais ou de lesões pré-ulcerativas nos pés de pacientes diabéticos com diminuição da sensibilidade por neuropatia diabética associada a pequenos traumas, geral mentecausadas por cal çados inadequados, dermatoses comuns, ou manipulações impróprias dos pés pelos pacientes ou por pessoas nãohabilitadas. As úlceras associadas à doença vascular periférica constituem uma menor parcela, porém requerem cuidados imediatos eespecial izados.

A detecção precoce do "pé em risco" pode ser feita facilmente pela inspeção e aval iação da sensi bi lidade através de testes si mples e de baixo custo ${ }^{19,20}$. 0 


\begin{tabular}{|c|c|c|}
\hline - neuropatia ausente & baixo risco & $\begin{array}{l}\text { - educação } \\
\text { - cuidados gerais } \\
\text { - avaliação anual }\end{array}$ \\
\hline - neuropatia presente & risco moderado & $\begin{array}{l}\text { - educação } \\
\text { - cuidados gerais } \\
\text { - sapatos adequados } \\
\text { - avaliação semestral }\end{array}$ \\
\hline $\begin{array}{l}\text { - neuropatia presente e/ou } \\
\text { deformidades, } \\
\text { - proeminências e/ou } \\
\text { isquemia, } \\
\text { - úlcera/amputação prévia }\end{array}$ & alto risco & $\begin{array}{l}\text { - educação } \\
\text { - cuidados gerais } \\
\text { - sapatos adequados e/ou } \\
\text { especiais } \\
\text { - seguimento por equipe } \\
\text { especializada } \\
\text { - avaliação trimestral }\end{array}$ \\
\hline
\end{tabular}

Quadro 5 - Cuidados gerais para os pés

1. Examine seus pés diariamente: se for necessário peça ajuda a um familiar ou use um espelho.

2. Avise seu médico se tiver calos, rachaduras, alterações de cor ou úlceras.

3. Vista sempre meias limpas preferentemente de lã ou algodão.

4. Calce apenas sapatos que não the apertem, preferencialmente de couro macio. Não use sapatos sem meias.

5. Sapatos novos devem ser usados aos poucos. Use-os nos primeiros dias apenas em casa por no máximo duas horas. Compre-os de preferência no final do dia.

6. Nunca ande descalço, mesmo em casa.

7. Laves seus pés diariamente, com água morna e sabão neutro. Evite água quente. Seque bem os pés, especialmente entre os dedos.

8. Após lavar os pés use um hidratante a base de lanolina, mas não aplique entre os dedos.

9. Corte as unhas de forma reta, horizontalmente.

10. Não remova os calos, nem procure corrigir unhas encravadas. Procure um tratamento profissional.

emprego dessas medi das e a educação de profissi onais, pacientes e familiares podem reduzir em até $50 \%$ o risco de amputação.

E mbora não existam dados populacionais sobre a prevalência das complicações crônicas do DM no Brasil, estima-se que o número de complicações crônicas seja elevado. Além disso, provavelmente apenas uma pequena fração da população dos pacientes diabéticos é avaliada regularmente para a presença de complicações nas suas fases iniciais e recebe orientação terapêutica apropriada.

Para reduzir o impacto dessas complicações na quali dade de vi da dos pacientes diabéti cos e prol ongar a sua vida de uma forma mais saudável e participativa na sociedade, delineou-se o projeto que a seguir está descrito.

\section{Objetivos}

- I mplantação e implementação de métodos para rastreamento da nefropatia e retinopatia diabéticas e do pé em risco para úlceras etratamento para as compli cações detectadas.

- Promover pesquisas operaci onais em determinados locais que desenvolvam o programa.

\section{MÉTODOS}

\section{Rastreamento das complicações}

População-al vo

O rastreamento das complicações crônicas será estimulado em todos os centros e locais que se dedicam ao atendimento de pacientes diabéticos. Serão utilizados métodos simples, de fácil emprego e de sensibilidade el evada.

Nos pacientes com DM tipo 1, o rastreamento para as complicações crônicas deve ser realizado cinco anos após o diagnóstico de DM ou cinco anos após a puberdade naqueles pacientes nos quais o DM surgiu antes de 12 ou 13 anos de idade. Em pacientes com DM tipo 2, o rastreamento deve ser feito por ocasi ão do diagnósti co de DM. E m pacientes com DM tipo 1 e ti po 2 o rastreamento deve ser repetido em intervalos de um ano. No caso da RD o rastreamento deve ser feito também quando houver sintomas dediminui ção da acuidade visual ou em toda paciente com DM tipo 1 logo no início da gravidez.

\section{Nefropatia Diabética}

O rastreamento deve iniciar com a real ização de exame comum de urina e urocultura em amostra casual de urina. $\mathrm{Na}$ ausência de hematúria e de infecção urinária, deve ser dosada a concentração de proteínas totais por método quantitativo na mesma amostra. 


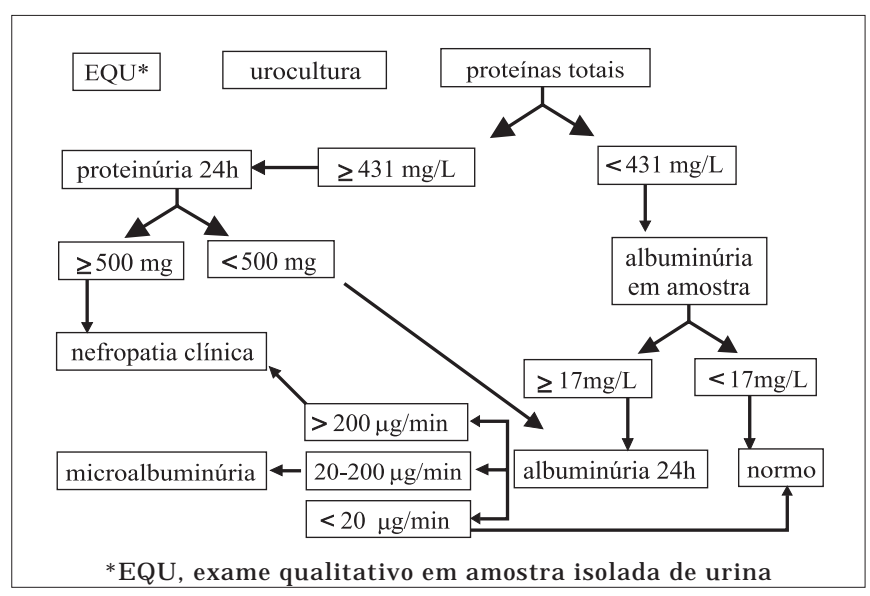

F ig. 1 - F l uxograma para o rastreamento ediagnósti co da Nefropatia Diabética

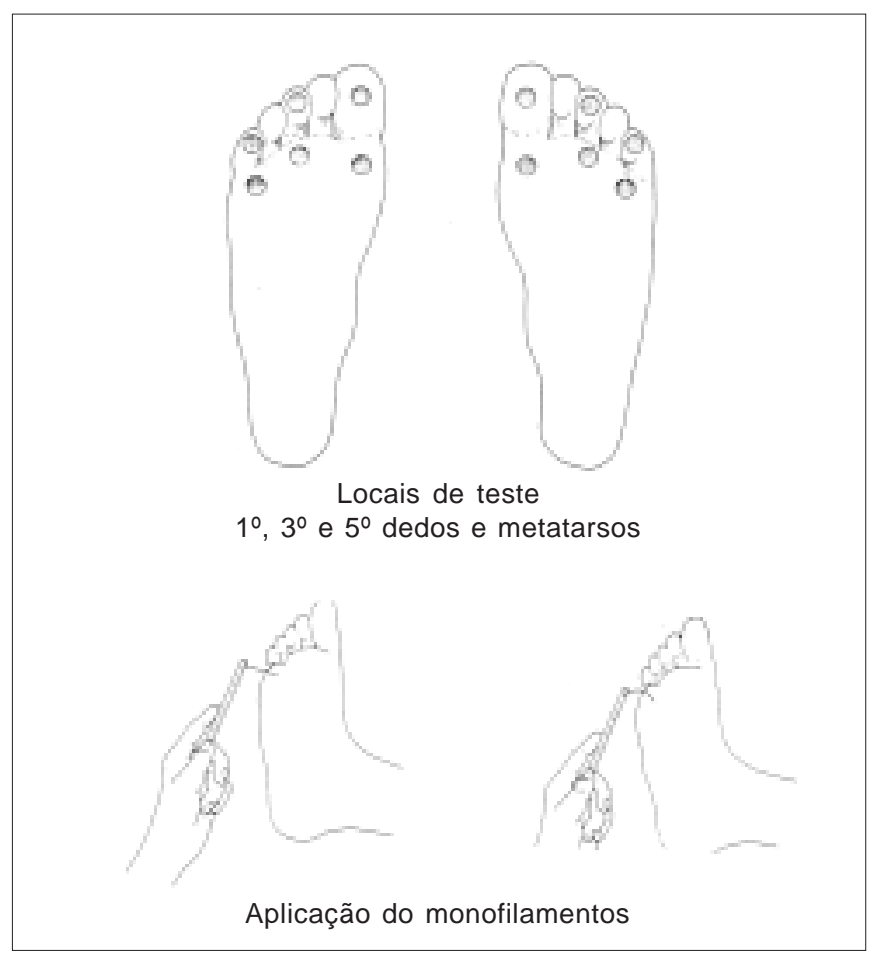

F ig. 3 - Técni ca deapl icação demonofi lamento epontos do pé que devem ser testados

Valores de proteinúria $430 \mathrm{mg} / \mathrm{L}$ apresentam uma sensibilidade de $100 \%$ e especificidade de $80 \%$ para o diagnóstico de nefropatia clínica. E ste diagnóstico deve ser confirmado com dosagem de proteínas totais em urina de 24 horas. Se o valor de proteínas totais na amostra casual de urina for $<430 \mathrm{mg} / \mathrm{L}$, real iza-se a medida de al buminúria na mesma amostra de urina. Val ores de al bumi núria ${ }^{3}$ 17 mg/L são considerados diagnósticos de microalbuminúria (nefropatia incipiente) e valores menores do que estes são considerados normais. A presença de microalbuminúria deve ser confirmada em urina de 24 horas com tempo marcado (registra-

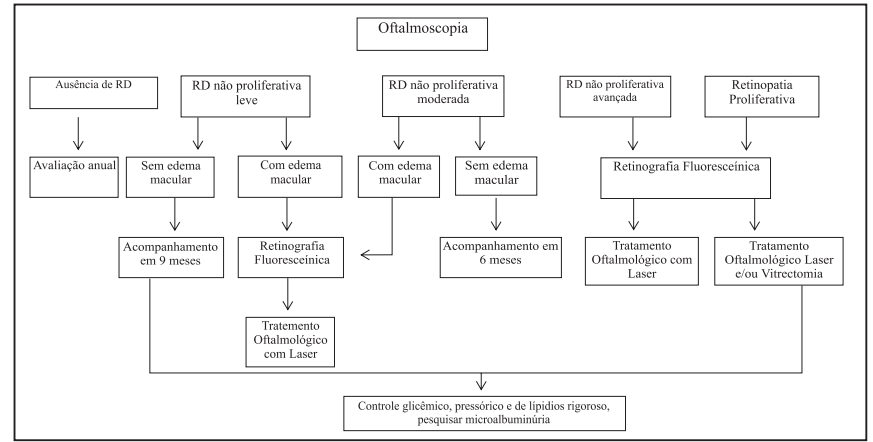

Fig. 2 - Fluxograma de diagnóstico de tratamento da Retinopatia Diabética (RD)

se o horário do início e do fim da col eta). (Fig. 1)

No Quadro 1 estão descritos os valores de albuminúria e de protei núria utilizados para o diagnóstico de ND9,10. A dosagem de al bumina na urina deve ser realizada através de método sensível e específico: imunoturbi dimetria (ITM) ou radioimunoensaio.

Devidoà grande variabilidade bi ol ógi ca da excreção urinária da albumina (EUA), é preciso confirmar o diagnóstico de microalbuminúria com uma medida em amostra isol ada e uma medi da de urina de $24 \mathrm{~h}$, real izadas com um intervalo de três a seis meses. Nos pacientes com diagnóstico de micro ou macroal bumi núria deve ser realizada a medida da creatinina sérica anualmente para avaliação da função renal.

\section{Retinopatia Diabética}

Idealmente a avaliação oftalmológica deve ser realizada por oftalmologista da rede pública. U ma al ternativa para os locais em que o acesso a oftalmologistas seria difícil, é a obtenção de imagens digitais em sistemas el etrônicos. Essas imagens, obtidas por técnicos treinados, seriam armazenadas em discos e posteriormente analisadas por especialistas. A constatação de qual quer grau de RD exige uma avaliação complementar que deverá ser realizada em Centros de Referências credenciados pela Sociedade Brasileira de Retina e Vítreo e Soci edade Brasileira de Diabetes, conforme descrito na Fig. 2.

\section{Pé em risco de úlceras}

Os fatores de risco mais importantes para o aparecimento de úl ceras nos pés são a neuropatia diabética periférica, a desinformação sobre os cui dados com os pés, presença de pontos de pressão anormal que favorecem as cal osidades, as deformidades, a doença vascular periférica eas dermatoses comuns (sobretudo entre os dedos). Os pacientes com história prévia de úlcera ou amputação são parti cularmente consi derados como de el evado risco para o desenvolvimento de novas úlceras. A avaliação clínica baseia-se em um exame físico dos 
pés que incl ui a inspeção e pal pação da pele (coloração e temperatura), unhas, do subcutâneo e da estrutura dos pés, a palpação dos pulsos arteriais tibial posterior e pedioso (que pode estar ausente em $10 \%$ de indivíduos normais) e a avaliação da sensibilidade protetora plantar. I dealmente, esta deve ser feita utilizando um monofilamento de $10 \mathrm{~g}$ em seis regiões do pé conforme demonstrado na Fig. 3 e de acordo com as instruções descritas no Quadro 2. Em cada consulta deverá ser realizada inspeção dos pés para avaliar a presença de unha encravada e/ou deformada, deformidades dos dedos e do arco plantar, áreas decal osidades, rachaduras, fissuras e bolhas, úlceras e intertrigo micótico. Além disso, deve-se real izar uma inspeção dos calçados, verificar a presença de pontos deatrito ou de pressão plantar excessiva, desgaste irregular, etc. Seguindo esses procedi mentos, os paci entes diabéticos em risco de apresentarem úl ceras poderão ser facilmente identificados (Quadros 3 e 4).

\section{E mprego de medidas de tratamento}

\section{Nefropatia}

Pacientes diabéticos tipo 1 com micro ou macroalbuminúria, mesmo quando forem normotensos, devem receber agentes ini bi dores da enzima de conversão da angiotensina (ECA) como primeira escol ha, respeitando-se as contra-indicações (gestação, hi percal emia, pacientes idosos com estenose bilateral da artéria renal ou com doença renal avançada $=$ creatinina sérica $>3,0 \mathrm{mg} / \mathrm{dl}$ ) e efeitos adversos (tosse, angioedema, urticária, leucopenia, perda do paladar). Nas mulheres em idade fértil deve-se alertar a cerca dos riscos destas drogas durante a gravidez. Nos pacientes diabéticos tipo 2 há al gumas evidências que suportem a sua indicação preferencial ${ }^{21}$, inclusive como prevenção do desenvol vimento da ND22. A mai oria dos casos de ND apresentarão também HAS. Havendo a opção pelos inibidores da ECA e não ocorrendo a redução dos níveis tensionais abaixo de $130 / 85 \mathrm{~mm}$ $\mathrm{Hg}$, acrescentam-se outros agentes. De fato estes pacientes necessitam de dois ou mais agentes para obter um bom controle pressórico. Pode-se iniciar com di uréti cos tiazídi cos em baixa dosagem (12,5 a 25 mg/dia), indapamida ou se houver insuficiência renal (creatinina sérica $>2,5 \mathrm{mg} / \mathrm{dl}$ ) furosemida, ou beta-bloqueadores, idealmente do tipo cardiossel etivos, desde que adequadamente consi deradas as contra-indicações e efeitos adversos, que limitam o seu uso no DM. Os agentes bloqueadores dos canais de cál cio devem ser utilizados com cautela em pacientes diabéticos, pois podem estar associados a um aumento da mortalidade por doença cardiovascular, quando usados isoladamente $\mathrm{e}^{23}$.
Portanto, recomenda-se evitar o seu emprego como monoterapia inicial, podendo utilizá-los quando não se tiver obtido o controle adequado dos níveis pressóricos com beta-bloqueadores, inibidores da ECA e diuréticos.

Os pacientes macroalbuminúricos devem receber prescri ção de dieta hi poprotéica $(0,8 \mathrm{gr}$ de proteína/kg de peso), ser avaliados pelo menos a cada três meses e ser feito todo o esforço para a obtenção de um bom controle glicêmico. Recomenda-se tratar intensamente os fatores de risco car diovascular associados, como por exemplo, a HAS, a dislipidemia e o tabagi smo. Outras complicações do DM, freqüentemente associadas, como a retinopatia, neuropatia, vasculopatia periférica e cardiopatia isquêmica devem ser pesquisados.

\section{Retinopatia}

Na presença de qualquer grau de RD, é crucial procurar obter o melhor controle possível da pressão arterial (<130/85 mm Hg), e da glicemia e do perfil li pídico. O tratamento oftal mológi co específico, de acordo com a linhas gerais do fluxograma em anexo, deverá ser realizado em Centros deR eferência capacitados (Figura 2).

\section{Pé em risco de úlceras}

A detecção de diminuição de sensibilidade ao monofilamento ou de insuficiência circulatória periférica, assim como a presença de lesões préul cerativas cutâneas ou estruturais, definem o paciente em risco para úlceras. Esses pacientes devem receber material informativo de educação (Quadro 5), avaliação freqüente, receber cui dados por profissional habilitado a cerca do uso de calçados adequados e acesso a um tipo de calçado especial, se houver deformidades.

\section{E stratégi as}

- Conscientizar a população e o poder público da importância das complicações crônicas através de campanha publicitária emjornais, televisão, cartazes publicitários (out-doors) nas princi pais cidades doPaís. - Sensibilizar e priorizar a detecção das complicações crôni cas nos Programas de E du cação e Controle de Diabetes no País.

- Divulgar as normas através de Revistas Científicas (AMB , ABE M), fol hetos dedivulgação, E ncontros Científicos. (Programas locais, Harvard-J oslin-SBD, Congressos Nacionais de Diabetes, Endocrinologia, Oftal mol ogia e Congressos de Clínica Médica).

- Sensibilizar os responsáveis pela política de saúde para o repasse de recursos necessários na implementação destas medidas através do Sistema Único de Saúde. 
- Estimular a realização periódica de oficinas para treinamento teórico-prático destinadas aos clínicos, enfermeiros e demais membros das equi pes de saúde.

- Buscar recursos específicos em agências de financiamento internacionais.

\section{AGRADECIME NTOS}

Aos Laboratórios Servier e Biobrás por terem patrocinado a reunião realizada em São Paulo no dia 18/07/98.

Redação do documento final: J orge Luiz Gross, Mirela J obim de Azevedo, Sandra Silveiro, Sandra Roberta Gouvea Ferreira, Domingos Malerbi, Hermelinda Cordeiro Pedrosa. Participantes do Consenso: Adriana Costa e Forti, Ana Teresa Mana G. SantoMauro, Antônio Carlos Lerário, Antônio Roberto Chacra, Antônio Rodrigues Ferreira, Bernardo Leo Wajchemberg, Ethel Spichler, Helena Schmid, Hermelinda Cordeiro Pedrosa, J orge Luiz Gross, Laercio J oel Franco, Laurenice Pereira Lima, Márcio Nehme, Maria Teresa Zanella, Marília de Brito Gomes, Mirela J obim de Azevedo, Roberto Abdala Moura, Sandra Roberta Gouvea Ferreira, Sandra Silveiro, Simão A Lottenberg, Domingos Malerbi, Ruy Lyra, Reine M. Chaves Fonseca, J osé Egídio Paulo de Oliveira.

\section{REFERÊNCIAS BIBLIOGRÁFICAS}

1. Malerbi DA, Franco LJ, and the Brazilian Cooperative Group on the Study of Diabetes Prevalence. Multicenter study of the prevalence of diabetes mellitus and impaired glucose tolerance in the urban Brazilian population aged 3069 yr. Diabetes Care 1992; 15 : 1.509-16.

2. Nathan DM, Meigs J, Singer DE. The epidemiology of cardiovascular disease in type 2 diabetes mellitus: how sweet it is ...or is it? The Lancet 1997; 350 (suppl 1) : 4-9.

3. Wang SL, Head J Stevens L, Fuller J H. Excess mortality and its relation to hypertension and proteinuria in diabetic patients. The World Health Organization multinational study of vascular disease in diabetes. Diabetes Care 1996; 19: 305-12.

4. UK Prospective Diabetes Study Group. Tight blood pressure control and risk of macrovascular and microvascular complications in type 2 diabetes: UKPDS 38. BMJ 1998; 317: 703-13.

5. Captopril Prevention Project (CAPPP) in hypertension baseline data and current status. Hansson L, Hedner T, Lindholm L, Niklason A, Luomanmaki K, Niskanen L, Lanke J, Dahl of B, de Faire U, Morlin C, Karlberg BE, Wester PO, Bjorck J E. Blood Press 1997; 6: 365-7.

6. Pyorälä K, Pedersen TR, Kjekshus J , Faergeman O, Olson Ag, Thorgerson G, The Scandinavian Simvastatin Survival Study Group. Diabetes Care 1997; 20: 614-20.

7. Gall MA, Rossing P, Skott $P$, et al. Prevalence of micro- and macroalbuminuria, arterial hypertension, retinopathy and large vessel disease in European type 2 (non-insulin-dependent) diabetic patients. Diabetologia 1991; 34: 655-61.
8. Bruno RM, Goldani J C . Informações pessoais obtidas junto ao Sistema de Informações Ambulatoriais do Sistema Único de Saúde, Secretaria de Saúde do Meio Ambiente do Estado do Rio Grande do Sul.

9. Zelmanovitz T, Gross J L, Oliveira J R, et al. The receiver operating characteristics curve in the evaluation of a random urine specimen as a screening test for diabetic nephropathy. Diabetes Care 1997; 20: 516-19.

10. Zelmanovitz T, Gross J L, Oliveira J , Azevedo MJ . Proteinuria is still useful for the screening and diagnosis of overt diabetic nephropathy. Diabetes Care 1998; 21:1076-9.

11. Kiberd BA, Kailash KJ . Screening to prevent renal failure in insulin dependent diabetic patients: an economic evaluation. BMJ 1995; 311: 1595-9.

12. Gazis A, Page SR. Microalbuminuria screening in the UK: are we meeting european standards?. Diabetic Medicine 1996; 13: 764-7.

13. Aiello LP, Gardner TW, King GL, et al. Diabetic retinopathy. Technical review. Diabetes Care 1998; 21: 143-56.

14. Bagga P, Verma D, Walton C, Masson EA, Hepburn DA. Survey of diabetic retinopathy screening services in England and Wales. Diabetic Medicine 1998; 15: 780-2.

15. Ryder REJ. Screening for diabetic retinopathy in the 21st century. Diabetic Medicine 1988; 15 : 721-722.

16. Spilchler ERS, Spichler D, Martins CSF, et al. Diabetic lower extremities amputation - Rio de J aneiro, BR.. Diabetologia 1998; 41(A 279) : 90-96.

17. Pedrosa HC. Pé Diabético: aspectos fisiopatológicos, tratamento e prevenção. Rev Bras Neurologia e Psiquiatria 1997; 1: 131-5.

18. Pedrosa HC, Nery ES, Sena FV, et al. O desafio do projeto Salvando o Pé Diabético. Terapêutica em Diabetes. Bol etim do Centro BD 1998; $19: 1-10$.

19. Armstrog DG, Lavery LA, Vela AS, Quebedeaux TL, Fleischhli JG. Choosing a practical screening instrument to identify patients at risk for diabetic foot ulceration. Arch Intern Med 1998; 158: 289-92.

20. Boulton AJ M, Gries FA, J ervell J Á. Guidelines for the diagnosis and outpatient management of diabetic peripheral neuropathy. Diabetic Medicine 1998; 15: 508-14.

21. Ravid M, Savin H, I utrin I, Bental T, Katz B, Lishner M. Long-term stabilizing effect of angiotensin-converting enzyme inhibition on plasma creatinine and on proteinuria in normotensive type 2 diabetic patients. Ann Intern Med 1998; 128: 982-88.

22. Ravid M, Brosch D, Levi Z, Bar-Dayan Y, Ravid D, Rachamani $R$. Use of enalapril to attenuate decline in renal function in normotensive, normoalbuminuric patients with type 2 diabetes mellitus. A randomized, controlled trial. Ann Intern Med 1993; 118: 577-81.

23. Estacio RO, J effers BW, Hiatt WR, et al. The effect of nisoldipine as compared with enalapril on cardiovascular outcomes in patients with non-insulin dependent diabetes and hypertension. N Engl J Med 1998; 338: 645-52. 